\title{
Trailing vortices in a free-surface flow
}

\author{
Tong Chen and Allen T. Chwang ${ }^{\text {a) }}$ \\ Department of Mechanical Engineering, The University of Hong Kong, Hong Kong
}

(Received 3 November 2000; accepted 8 November 2001)

\begin{abstract}
The free-surface effect on two-dimensional flow structures, especially on the trailing vortices, is investigated numerically in this paper. The solution procedure employs a higher-order semi-implicit projection method. The numerical results have been compared with experimental data on wave elevation disturbed by a submerged translating hydrofoil with a positive angle of attack. The schematic computations reveal some interesting and unique characteristics of the trailing-vortex development in a laminar flow beneath a free surface. Based on the computational data, an empirical Strouhal-Reynolds-Froude number relationship is proposed for the purely laminar vortex shedding in a free-surface flow. Moreover, the numerical findings also indicate that the presence of a free surface accelerates the Strouhal vortex frequency, yet delays not only the onset of a large-scale Kármán instability but also the occurrence of transitional behaviors. The shedding frequency downshifts drastically or even becomes discontinuous when the secondary vortex grows on the suction side. A metastable transition frequency emerges at the same time and becomes distinct with increasing Reynolds number. The variation of the vortex-shedding Strouhal number with respect to both Reynolds and Froude numbers is obtained. The power spectra of the trailing wake consist of harmonics of the vortex-shedding and transition frequencies $\left(m f_{s}, n f_{t}\right)$, as well as their combinations $\left(m f_{s} \pm n f_{t}\right)$. (C) 2002 American Institute of Physics. [DOI: 10.1063/1.1432320]
\end{abstract}

\section{INTRODUCTION}

The transformation between kinetic and potential energies and the coexistence of viscous, gravity and surface tension forces at an unknown wavy boundary make the freesurface phenomena difficult to study. Wave signatures are known to be caused by the interaction between the vortexinduced wake behind a moving object and a deformable airwater interface. They are responsible for some interesting vortex behaviors, such as the vortex rebounding, rollers at wave crests, creation of secondary eddies with mass and momentum transport, straight and sharp surface depression labeled as scars and striations, and the wave resonance due to jet attachment to the free surface (Sheridan, Lin, and Rockwell ${ }^{1}$ ). A better understanding of these flow behaviors is fundamental to ocean engineering and undersea technology. However, the physics of the flow structures and the freesurface interaction, especially the Kármán instability of a trailing wake, remains elusive due to the complexity in devising and using mathematical formulations, numerical schemes and physical experiments (Sarpkaya $\left.{ }^{2}\right)$. Available experiments have shown that the trailing vortices can become inherently unstable to centrifugal and helical disturbances and to the existence of a wake-like or jet-like velocity profile. The primary vortices may also rebound due to shear and small eddies at the free surface (Ohring and Lugt ${ }^{3}$ ). Direct numerical solutions usually can not be avoided due to the complexity of water waves with the introduction of bodies

\footnotetext{
a) Author to whom correspondence should be addressed. Telephone: (852) 2859-2634; Fax: (852) 2858-5415. Electronic mail: atchwang@hkucc.hku.hk
}

into the wave field. The present prevailing numerical methods based on volume-discretization, as summarized by Tsai and Yue, ${ }^{4}$ is the boundary-fitted finite-difference method. With some simplifications on the free-surface boundary conditions (BC's), Zhang, Shen, and Yue ${ }^{5}$ had recently investigated the mechanism of vortex connection at a free surface in a three-dimensional (3D) numerical wave tank. Through an ascending vortex ring, they qualitatively confirmed the viscous and blockage double sublayers beneath the free surface and imitated the perpendicular trend of vortices when approaching a free-deformable boundary. Systematic computations indicated that the blockage layer results from the kinematic BC at the free surface, and the viscous surface layer is caused by the dynamic stress-free conditions. Meanwhile, large-eddy simulations for free-surface channel flows were also formulated by Hodges and Street ${ }^{6}$ with the emphasis on computational aspects and engineering applications. By visualizing the wake states of a flow past a cylinder close to the free surface, Sheridan, Lin, and Rockwell ${ }^{1}$ experimentally studied the wake instability, the Froude-number effect, the free-surface distortion, and the vorticity flux from the free surface and the cylinder surface. They concluded that the localized separation or complete separation from the free surface is responsible for the generation of a vorticity layer from the free surface. For underwater disturbances moving steadily at a transcritical velocity in shallow water channels, the bifurcation phenomenon of a succession of upstreamadvancing solitary waves may be excited. Zhang and Chwang $^{7}$ numerically investigated the viscous effect on these periodically generated solitons.

The physical similarity constraint in the free-surface flow has seriously limited the physical experiments for para- 


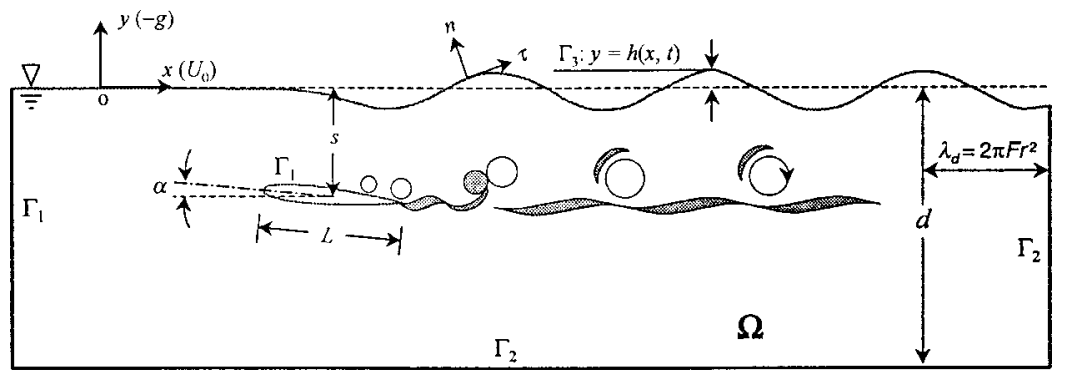

FIG. 1. Physical domain for a fully submerged hydrofoil in a uniform flow.

metric studies. In order to implement the fully nonlinear free-surface BC's and to efficiently tackle complex configurations, a numerical finite element procedure was formulated for 3D incompressible free-surface flows by Chen, Chwang, and Zhang. ${ }^{8}$ The central interest of the present work lies in the study of the generation of trailing vortices in the wake of a fully submerged two-dimensional (2D) hydrofoil. By monitoring the alternation behaviors of a trailing wake, the freesurface effect on the flow structure is systematically examined in the numerical experiments, involving substantial combinations of Reynolds and Froude numbers $(\mathrm{Fr}=0.0-$ 0.62 and $\operatorname{Re}=3000-30000)$.

\section{DESCRIPTION OF THE FLOW PROBLEM}

Let us consider a fully submerged hydrofoil in a uniform flow, and focus on the free-surface influence on the entire flow structure, particularly on the trailing vortices. In a $2 \mathrm{D}$ Cartesian reference frame $\mathbf{x}=(x, y)$ shown in Fig. 1 with $x$ axis in the uniform flow direction on the mean free surface and the $y$ axis pointing vertically upward, the dimensionless incompressible Navier-Stokes and continuity equations are

$$
\begin{aligned}
& \mathbf{u}_{t}+(\mathbf{u} \cdot \nabla) \mathbf{u}=-\nabla P+\operatorname{Re}^{-1} \nabla^{2} \mathbf{u}, \\
& \nabla \cdot \mathbf{u}=0,
\end{aligned}
$$

where the hydrodynamic pressure $P=p+y / \mathrm{Fr}^{2}$ is the total pressure $p$ minus the hydrostatic pressure, $\mathbf{u}=(u, v)$ is the velocity and $t$ is the time.

$$
\operatorname{Fr}=U_{0} /(g L)^{1 / 2} \text { and } \operatorname{Re}=\rho U_{0} L / \mu_{0},
$$

are the Froude and Reynolds numbers, respectively, based on a characteristic length $L$, a reference velocity $U_{0}$, the molecular viscosity $\mu_{0}$, the gravitational constant $g$ and the fluid density $\rho . p$ is nondimensionalized by $\rho U_{0}^{2}$.

The governing equations are to be solved subject to the following BC's: (a) essential type of specified velocity on solid surfaces (no-slip) and inlet (incident free-stream) $\Gamma_{1}$ and (b) natural type of prescribed traction vector at open boundaries (traction free) $\Gamma_{2}$ truncated far enough from the region concerned and at a time-dependent free surface $\Gamma_{3}$ defined by $y=h(x, t)$

$$
\begin{aligned}
& \left.\mathbf{u}\right|_{\Gamma_{1}}=\mathbf{b}, \\
& \left.(-p \mathbf{I}+\boldsymbol{\sigma}) \cdot \mathbf{n}\right|_{\Gamma_{2}+\Gamma_{3}}=\mathbf{c} .
\end{aligned}
$$

Here, $\boldsymbol{\sigma}=\operatorname{Re}^{-1}\left[\nabla \mathbf{u}+(\nabla \mathbf{u})^{\mathrm{T}}\right]$ is the deviatoric stress, $\mathbf{I}$ is the identity tensor and superscript " $T$ " denotes the transposed tensor. $\mathbf{n}=\left(n_{x}, n_{y}\right)$ and $\boldsymbol{\tau}=\left(n_{y},-n_{x}\right)$ are, respectively, the outward unit vectors normal and tangential to the surfaces. The fact that no fluid particle leaves the free surface provides additionally the kinematic BC

$$
h_{t}+u h_{x}=v \quad \text { on } \Gamma_{3} .
$$

Meanwhile, the traction is no longer free if the external freesurface tension $q$ is appreciable: $\mathbf{c}=q \mathbf{n}$. Assuming that $q$ is proportional to the interface curvature

$$
\kappa=h_{x x}\left(1+h_{x}^{2}\right)^{-3 / 2},
$$

we have

$$
q=\mathrm{We}^{-1} \kappa,
$$

where $\mathrm{We}=\rho U_{0}^{2} L / \gamma$ is the Weber number, $\gamma$ being the coefficient of surface tension. Introducing vorticity $\omega=v_{x}-u_{y}$, dynamic condition (4) at $\Gamma_{3}$ can be rewritten as

$$
\begin{aligned}
& \left.\frac{2}{\operatorname{Re}} \frac{\partial u}{\partial n}\right|_{\Gamma_{3}}=(p+q) n_{x}-\frac{\omega}{\operatorname{Re}} n_{y}, \\
& \left.\frac{2}{\operatorname{Re}} \frac{\partial v}{\partial n}\right|_{\Gamma_{3}}=(p+q) n_{y}+\frac{\omega}{\operatorname{Re}} n_{x},
\end{aligned}
$$

which also implies the relations

$$
\begin{aligned}
& \frac{2}{\operatorname{Re}} \frac{\partial u_{n}}{\partial n}=p+q, \\
& 2 \frac{\partial u_{\tau}}{\partial n}=-\omega,
\end{aligned}
$$

by eliminating normal forces $(p+q)$ and vorticity $\omega$, respectively, where $u_{n} \equiv \mathbf{u} \cdot \mathbf{n}$ and $u_{\tau} \equiv \mathbf{u} \cdot \boldsymbol{\tau}$ are the normal and tangential velocity components, and

$$
\frac{\partial u_{n}}{\partial n}=\left(1+h_{x}^{2}\right)^{-1}\left[u_{x} h_{x}^{2}-\left(v_{x}+u_{y}\right) h_{x}+v_{y}\right] .
$$

It is of interest to note that dynamic $\mathrm{BC}$ (7) reduces to a kinematic one $(8 b)$ along the tangential direction. For a steady-state free surface problem, $(8 b)$ turns out to be

$$
\omega=-2 u_{p} \kappa,
$$

where $u_{p}$ denotes the particle speed at the free surface (Longuet-Higgins ${ }^{9}$ ).

Proper initial conditions and solvability constraints should be imposed for outflow problems to guarantee the existence of a solution (Gresho and $\mathrm{Sani}^{10}$ ). 


\section{COMPUTATIONAL ISSUES}

The mathematical model is numerically realized by the Galerkin-weighted residual method. The computational domain $\Omega$ is discretized with a finite number of isoparametric quadrilateral elements. In each element, the variables $(\mathbf{x}, \mathbf{u}, P)$ are approximated by the equal-order trial function $\Psi$ in terms of their transient nodal values. With the second-order CrankNicolson time integration, standard Galerkin's procedure turns Eqs. (1) and (2) into an algebraic system as

$$
\begin{aligned}
& \mathbf{M} \mathbf{u}^{n+1}+\mathbf{G} \Phi^{n+1} \\
& =\mathbf{M} \mathbf{u}^{n}-\mathbf{G} \Phi^{n}-\Delta t\left[\left(\mathbf{C}+\operatorname{Re}^{-1} \mathbf{K}\right) \mathbf{u}-\mathbf{F}\right]^{n+1 / 2}, \\
\mathbf{G}^{\mathrm{T}} \mathbf{u} & =0,
\end{aligned}
$$

symbolically equivalent to

$$
\left(\begin{array}{cc}
\mathbf{M} & \mathbf{G} \\
\mathbf{G}^{\mathrm{T}} & \mathbf{0}
\end{array}\right)\left(\begin{array}{c}
\mathbf{u}^{n+1} \\
\mathbf{\Phi}^{n+1}
\end{array}\right)=\left(\begin{array}{c}
\mathbf{r} \\
0
\end{array}\right)
$$

where

$$
\Phi=\Delta t P / 2
$$

and

$$
\begin{aligned}
& \mathbf{M}=\int_{\Omega} \Psi \Psi^{\mathrm{T}} d \Omega, \quad \mathbf{G}=\int_{\Omega} \Psi \nabla \Psi^{\mathrm{T}} d \Omega, \\
& \mathbf{K}=\int_{\Omega} \nabla \Psi \nabla \Psi^{\mathrm{T}} d \Omega, \quad \mathbf{C}=\left(\mathbf{u}-\mathbf{u}^{g}\right)^{e} \cdot \mathbf{G}, \\
& \mathbf{F}=\int_{\Gamma_{2}+\Gamma_{3}} \frac{1}{\operatorname{Re}} \frac{\partial \mathbf{u}}{\partial n} \Psi d \Gamma, \quad \mathbf{u}^{g}=\left(\mathbf{x}^{n+1}-\mathbf{x}^{n}\right) / \Delta t .
\end{aligned}
$$

Here, superscripts " $n+1$ " and " $n$ " refer to the current and previous time levels, $\Delta t$ is the time increment and superscript " $e$ " denotes the averaged values taken at element centroids. $\mathbf{K}$ is the Laplacian matrix and $\mathbf{G}$ the gradient matrices. The nonlinear convective matrix $\mathbf{C}$ has been modified to incorporate the mesh velocity $\mathbf{u}^{g}$ since the mesh has to be adaptive to the change of topology. To keep the procedure efficient by avoiding the global storage and an implicit solution, the consistent mass matrix $\mathbf{M}$ is lumped onto the diagonals, and penalized to impose the essential $\mathrm{BC}(3)$. The traction BC (4) and the dynamic BC (7) have been included in the boundary integrals of $\mathbf{F}$. To reduce the computational cost, the coefficient matrices are generated using the reduced-order integration based on the midpoint Gaussian quadrature.

Equation (11) can be decomposed into

$$
\left(\begin{array}{cc}
\mathbf{M} & \mathbf{0} \\
\mathbf{G}^{\mathrm{T}} & -\mathbf{G}^{\mathrm{T}} \mathbf{M}^{-1} \mathbf{G}
\end{array}\right)\left(\begin{array}{cc}
\mathbf{I} & \mathbf{M}^{-1} \mathbf{G} \\
\mathbf{0} & \mathbf{I}
\end{array}\right)\left(\begin{array}{c}
\mathbf{u}^{n+1} \\
\mathbf{\Phi}^{n+1}
\end{array}\right)=\left(\begin{array}{c}
\mathbf{r} \\
0
\end{array}\right),
$$

leading to the fractional projection scheme

$$
\begin{aligned}
& \mathbf{M} \mathbf{u}^{*}=\mathbf{r}, \\
& \mathbf{G}^{\mathrm{T}} \mathbf{M}^{-1} \mathbf{G} \Phi^{n+1}=\mathbf{G}^{\mathrm{T}} \mathbf{u}^{*}, \\
& \mathbf{u}^{n+1}=\mathbf{u}^{*}-\mathbf{M}^{-1} \mathbf{G} \Phi^{n+1},
\end{aligned}
$$

in which $\mathbf{u}^{*}$ is an intermediate velocity. The pressure and velocity are thus uncoupled based on the conservation of mass. The above procedure may also be interpreted by the Hodge decomposition principle that a divergent-free velocity field $\mathbf{u}$ (i.e., $\nabla \cdot \mathbf{u}=0$ ) can always be constructed by subtracting the curl-free velocity $\nabla \Phi$ from any given $\mathbf{u}^{*}$, as long as $\nabla^{2} \Phi=\nabla \cdot \mathbf{u}^{*}$. Bearing in mind that coefficient matrix $\mathbf{G}^{\mathrm{T}} \mathbf{M}^{-1} \mathbf{G}$ is a discrete approximation of the Laplacian operator, Poisson equation (13b) is practically replaced by

$$
\mathbf{K} \Phi^{n+1}=\left(\mathbf{u}^{*}\right)^{\mathrm{T}} \mathbf{G}+\mathbf{B},
$$

where by recalling $(13 \mathrm{c})$, we have

$$
\mathbf{B}=\int_{\Gamma_{1}+\Gamma_{2}}\left(\nabla \Phi-\mathbf{u}^{*}\right) \cdot \mathbf{n} \Psi d \Gamma=-\int_{\Gamma_{1}+\Gamma_{2}} \mathbf{u}^{n+1} \cdot \mathbf{n} \Psi d \Gamma .
$$

Equation (14) is also subject to the Dirichlet-type constraint of $\Phi$ on the free surface by dynamic BC (8a)

$$
\left.\Phi\right|_{\Gamma_{3}}=\frac{\Delta t}{2}\left(\frac{h}{\mathrm{Fr}^{2}}-\frac{\kappa}{\mathrm{We}}+\frac{2}{\operatorname{Re}} \frac{\partial u_{n}}{\partial n}\right) .
$$

The weak formulations (10a) and (14) are obtained by the Green identity through integration by parts, respectively. $\mathbf{B}$ is a source vector imposing the natural BC's for $\mathbf{u}$ in the normal direction. It is worth pointing out that if the calculation is for a nonfree-surface flow, no pressure BC is required. This is consistent with the original Navier-Stokes equations that only need a reference pressure datum. In the present numerical procedure, only Eq. (14) needs to be solved implicitly (using the diagonally preconditioned conjugate gradient solver), and only the symmetric and definite $\mathbf{K}$ requires global storage. The data structure is specially designed for fast large-domain and free-surface applications. Being crucial to the present approach, $\mathbf{K}$ is stored in a symmetric triad row format with pre-located pointers. This format geometrically produces a half "bandwidth" not exceeding the half maximum number of neighboring nodes and irrelevant to any node numbering. The latter feature makes the grid refinement by automatic cell bisection very straightforward and much easier. Two sub-iterations are carried out to obtain the midtime-level " $n+1 / 2$ " values.

Fully nonlinear kinematic BC (5) is implemented to update the free surface in a time-marching manner. To keep the global second-order accuracy in time, the Crank-Nicolson finite difference scheme is adopted while the convective term is treated with the third-order upwind scheme. Artificial damping is used at the up/downstream open boundaries for outgoing waves. The damping length is $\lambda_{d}=2 \pi \mathrm{Fr}^{2}$ from the linear theory. The discretized form thus becomes

$$
a h_{i-2}^{n+1}+b h_{i-1}^{n+1}+c h_{i}^{n+1}+d h_{i+1}^{n+1}=e,
$$

where

$$
\begin{aligned}
& a=d / 2, \quad b=-3 d, \quad c=1+3 d / 2+W \Delta t, \\
& d=u_{i}^{n+1 / 2} \Delta t / \Delta x, \\
& e=h_{i}^{n}-\Delta t\left(\Delta h^{n} / 2 \Delta x-v_{i}^{n+1 / 2}\right), \\
& \Delta h=2 h_{i+1}+3 h_{i}-6 h_{i-1}+h_{i-2},
\end{aligned}
$$



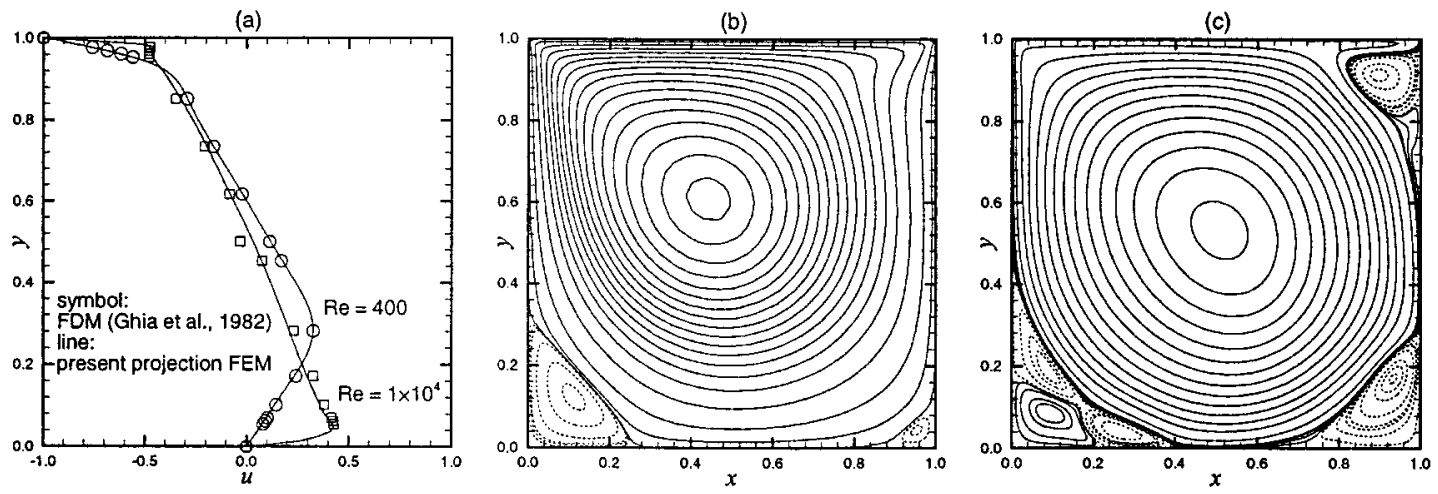

FIG. 2. Benchmark validation by the shear-driven cavity flow, (a) $u$-velocity component along central line $x=0.0$ and streamlines for (b) Re $=400$ and (c) $\operatorname{Re}=1 \times 10^{4}$ (FEM meshes: original total nodes $=6409$ and total elements $=6168$; refined total nodes $=25153$ and total elements $=24672$ ).

$$
\Delta x=2 x_{i+1}+3 x_{i}-6 x_{i-1}+x_{i-2} .
$$

$W(x)$ is a quadratic damping function of the free-surface waves designed by Israeli and Orszag. ${ }^{11}$ Subscript $(i-1)$ refers to the upstream node and $(i+1)$ the downstream one. For this purpose, the free-surface grid numbering is bubblesorted for the present unstructured mesh. Equation (17) is to be solved by the quadra-diagonal Gauss elimination without any wave-profile filtering or smoothing. For details, refer to Chen and Chwang. ${ }^{12}$ A field absorber (Lungu and Mori ${ }^{13}$ ) is adopted at the downstream exit for velocity fluctuations due to the wave and wake motions. The mesh adaptation employed is the Laplacian smoothing scheme. It is done by shifting each interior node to the center of the surrounding polygon and is only applied to the upper part of the domain adjacent to the free surface.

\section{RESULTS AND DISCUSSION}

\section{A. Model validations}

The present 2D numerical formulation is a direct dedimensionalization of the 3D code (Chen, Chwang, and Zhang $^{8}$ ) for simulating the free-surface flow past a vertical circular cylinder. By switching off the free-surface option, the nonfree-surface part of the 2D code is first validated by the benchmarks of a shear-driven cavity flow and the flow past a circular cylinder. The solution efficiency is enhanced by the multigrid acceleration technique based on the automatic cell bisection. For various Reynolds numbers (Re $=400-10000)$ in the cavity flow, excellent agreement has been reached with the higher-resolution $\left(257^{2}\right)$ finite difference calculations (Ghia, Ghia, and $\mathrm{Shin}^{14}$ ) for velocity, pres-
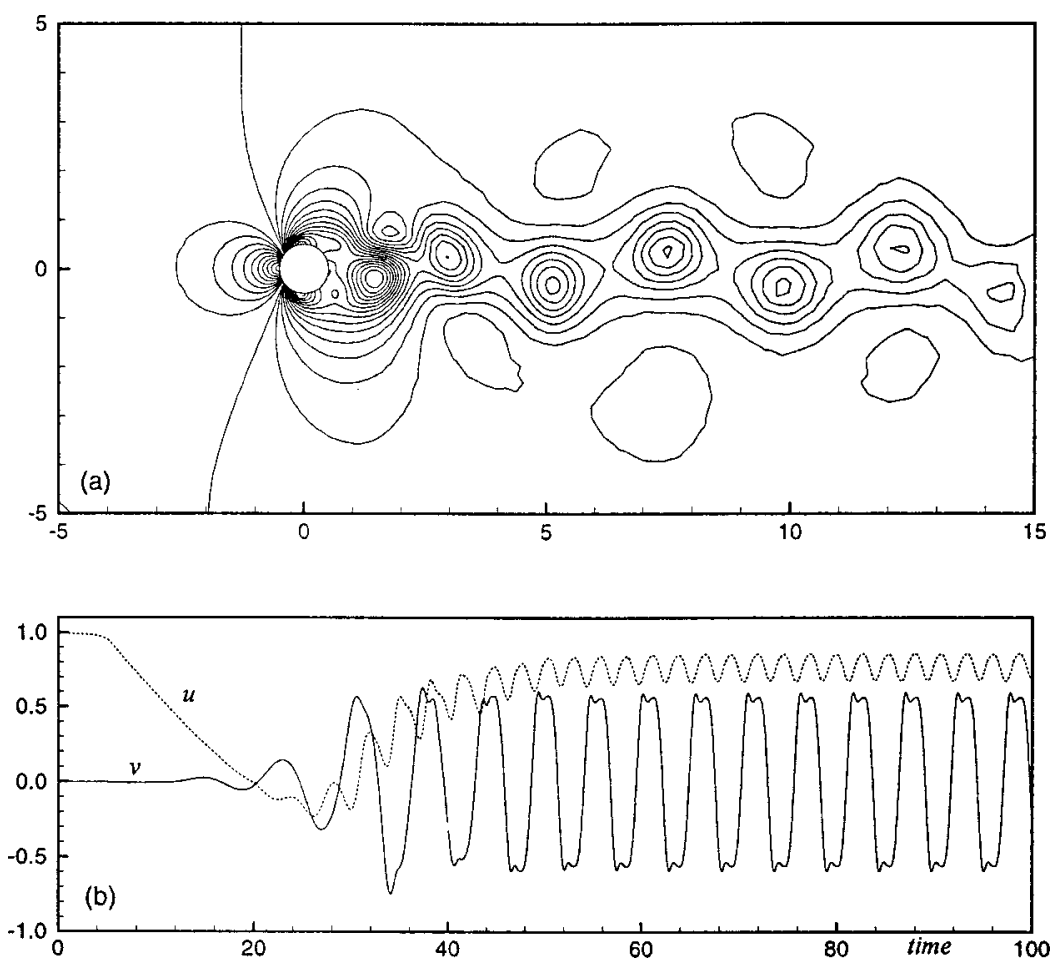

FIG. 3. Benchmark validation by the flow past a circular cylinder for $\mathrm{Re}=150$, (a) instantaneous pressure contours $\left(P_{\min }=-0.7, P_{\max }=0.6, \Delta P=0.05\right)$ and $(\mathrm{b})$ velocity signals recorded at sampling point $(5.0,0.0)$. Mesh refined when the periodicity of trailing vortices starts to develop $(t>40)$. 


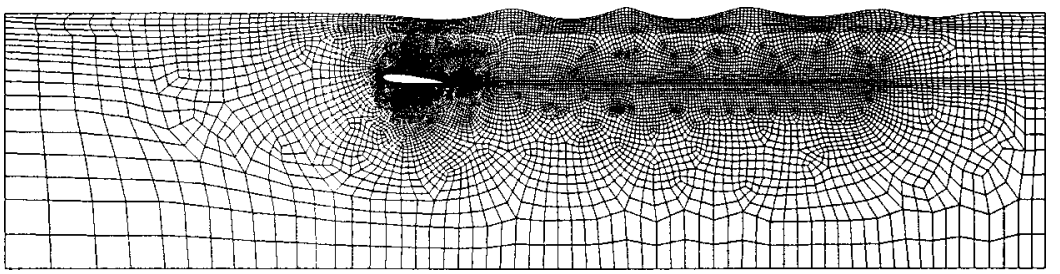

FIG. 4. Initial mesh division (total nodes $=15324$, total elements $=15076$, free-surface nodes $=209$ and bodysurface element $=184$ ). The one-level refinement of cell bisection gives total nodes $=60800$, total elements $=60304$, free-surface nodes $=417$ and body-surface elements $=368$.

sure and vorticity distributions, emphasizing the capacity of the present method for capturing the primary and secondary flow details. Typical cases are presented in Fig. 2 for the vertical profiles of $u$-velocity component along the central line and the streamlines for different Reynolds numbers. For the flow past a circular cylinder, Fig. 3(a) shows the instantaneous pressure contours at $\mathrm{Re}=150$. The recorded velocity signals $(u, v)$ at sampling point $x=5.0$ and $y=0.0$ are shown in Fig. 3(b). The Fourier transform of the signals from $t$ $=50.0$ to 100.0 (when the periodicity has been fully developed) results in a vortex shedding Strouhal number St $=0.183$, identical to the experimental measurement of Williamson $^{15}$ up to the third decimal place. Similarly, for $\mathrm{Re}=120, \mathrm{St}=0.175$ is obtained, which is very close to the experimental measurement of 0.173 . It is noted that the computational domain is restrictive and not wide enough to eliminate the sidewall effect. Nevertheless, the vortex shedding behavior is very well demonstrated for the present validation purpose.

To further validate the present code, the computed freesurface elevations are compared with experimental data of Duncan. ${ }^{16}$ The experiment conducted by Duncan ${ }^{16}$ was primarily to explore the wave-breaking criterion and the wake of the hydrofoil was ignored. The computational conditions for a free-surface flow are set to approximately reproduce the experiment of Duncan ${ }^{16}$ for a fully submerged NACA0012 hydrofoil in a uniform flow $\left(\mathrm{Fr}=0.5672, \mathrm{Re}=1.624 \times 10^{5}\right.$, and $\mathrm{We}=1784)$, in which no wave-breaking and bottom effect were observed. The depth of submergence $s=1.034$ is measured at the mid-chord and the angle of attack is $\alpha=5^{\circ}$. Figure 4 shows the computational domain $x(-6.5,10.0)$ and $y(-4.0, h)$, and the initial mesh division. The fine mesh is a one-level refinement by cell-bisection to the initial one for high Reynolds numbers. The time increment is $\Delta t=0.002$.

The simulation has to be time-accurate since the trailing vortex wake behind the hydrofoil is periodically shedding and inevitably forms a cooperative system with the freesurface wave motion. Therefore, the computed wave profiles are time-averaged $(t=15.0-26.0)$ in order to compare with experimental measurements, although the deviations are very small. The corroboration is evident as shown in Fig. 5, except for the minor under-prediction of the first wave trough right above the hydrofoil. This is owing to the fact that the Reynolds numbers computed are still far lower than the experimental one by which a fully developed turbulent wake is expected. The systematic decrease of viscosity clearly indicates that the viscosity has a stronger impact on wave amplitude than on wave phase for slender bodies such as a NACA0012 hydrofoil. Nevertheless, this may be misleading for blunt bodies, for which strong flow separation and jetflow effect may modify the wave dispersion to render the phase celerity sensitive as well.

\section{B. Flow structure in the presence of a free surface}

To investigate the free-surface effect on the flow structure, particularly on the alternation features of the trailing wake, parametric studies through numerical experiments are extensively conducted. Three Froude numbers $\mathrm{Fr}=0.0$, $0.5672(0.5578)$, and $0.6200(0.6097)$ are examined over a range of $\mathrm{Re}=0.3 \times 10^{4}-3.0 \times 10^{4}$. The numbers in parentheses are the converted Froude numbers

$$
\mathrm{Fr}_{s}=U_{0} /(g s)^{1 / 2}=(L / s)^{1 / 2} \mathrm{Fr}
$$

based on the submergence $s$. The coefficient of free-surface tension is fixed for $\mathrm{We}=1784$ in all the cases.

Figure 6 shows the instantaneous vorticity contours for $\mathrm{Re}=2.0 \times 10^{4}, \mathrm{Fr}=0.5672$, and $\mathrm{We}=1784$ in the vortex formation region $(t=26.0)$. Two major eddies with clockwise recirculation are generated in the shear layer between the upper suction side of the hydrofoil and the outer free stream. The secondary eddy $\left(e_{2}\right)$ will merge with the primary one $\left(e_{1}\right)$ before they leave the body surface together. It is found later that the strong nonlinear interaction between these two eddies results in drastic downshift or even discontinuity of the wake Strouhal number ( $\mathrm{St}$ ) and the emergence of an independent transition frequency. Small-scale eddies have emerged in the "dead water" area, climbing up along the body surface against the flow and pushing the separation points to the forward stagnation point. No recirculation is

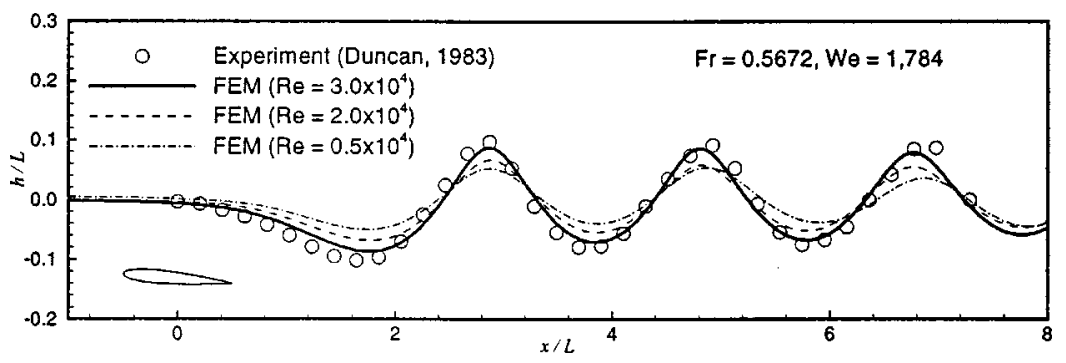

FIG. 5. Time-averaged wave profiles generated by a NACA0012 hydrofoil. 


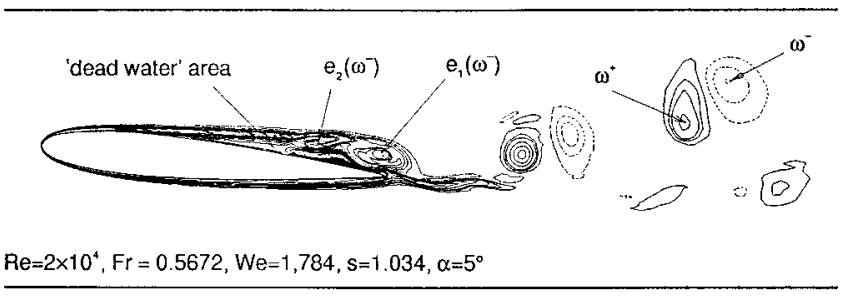

FIG. 6. Instantaneous vorticity contours in the vortex formation region around a NACA0012 hydrofoil $(t=26.0)$.

formed under the hydrofoil pressure side due to the positive angle of attack. Since the hydrofoil submergence is relatively deep for this Fr, the jet-flow effect between the upper body surface and the overhead free surface is not so obvious as observed by Sheridan, Lin, and Rockwell ${ }^{1}$ for flows past a horizontal circular cylinder very close to the free surface.

Figure 7 shows three typical vortex patterns for the cases indicated in the figure caption. The vorticity with strength $|\omega| \geqslant 0.5$ is flooded with light and dark colors for negative and positive vortices, respectively. For moderate $\mathrm{Re}=0.5$ $\times 10^{4}$ [see Fig. 7(a)], the flow remains purely laminar and the Kármán vortex street demonstrates periodical alternation with respect to the mean flow direction in a quasi-steady manner. The counter-rotating vortices are quite uniform and the incidence angle of flow plays an insignificant role. For an intermediately high $\operatorname{Re}=1.1 \times 10^{4}$ [see Fig. 7(b)], the flow reaches a transcritical stage and the transition is about to occur. The streamwise positive and negative vortices are twisted together. The unbalanced generation of shear due to the nonzero angle of attack has made positive vortices inclined. Such inclination renders the penetration and separation of negative vortices by the positive ones. As expected, the free-surface elevation gets higher as the undergoing disturbance gets stronger. Transition has already occurred at $\operatorname{Re}=3.0 \times 10^{4}$ [see Fig. 7(c)]. The counter-clockwise vortices $\left(\omega^{+}\right)$are streamwisely stretched out and connected like "flying" patches below the clockwise ones $\left(\omega^{-}\right)$, while the latter still try to retain a circular form and tend to ascend toward the free surface in the far downstream. It is of interest to note from Fig. 7(c) (also from Fig. 6) that the clockwise vortices, in this transition stage, are always accompanied by another counter-rotating one to form vortex pairs.

Figure 8 shows the instantaneous contours of the hydrodynamic pressure $\left(P=p+y / \mathrm{Fr}^{2}\right)$ corresponding to the previous cases, respectively. The pressure contour lines are plotted for $P \in[-0.5,0.5]$ with an increment $\Delta P=0.025$. The whirled low-pressure spots locate the vortex centers. However, as the stretch of $\omega^{+}$for higher Reynolds numbers [see Fig. 7(c)], the vortex cores are too vague to discern for the level of pressure contours plotted. Three small regions where the pressure $\left(P_{\min } \cong-1.0\right)$ exceeds the negative pressure plot-limit can be found in Fig. 8(c) at the upper leading edge and the two major eddy cores near the trailing edge.

Figures 9(a)-9(d) show the computed field variables of velocity $u$, velocity $v$, dynamic pressure $P$ and vorticity $\omega$, probed along a vertical line passing through the first wave crest. These figures clearly depict the blockage sublayer adjacent to the free surface due to the kinematic free-surface BC. The viscous sublayer is not so obvious under the first wave crest, although a very thin vortex-sheet attachment (thickness $\delta \cong 0.043$ ) with counter-clockwise vorticity $\left(\omega^{+}\right)$ can be observed from Fig. 9(d) right below the free surface when $\operatorname{Re} \rightarrow 3.0 \times 10^{4}$. The free-surface vortex sheet grows along the free surface, forming a thin viscous sublayer and a

(a) $\operatorname{Re}=0.5 \times 10^{4}$

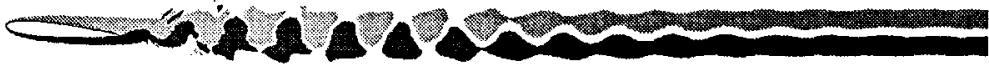

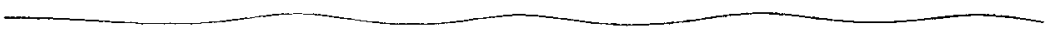

(b) $R e=1.1 \times 10^{4}$
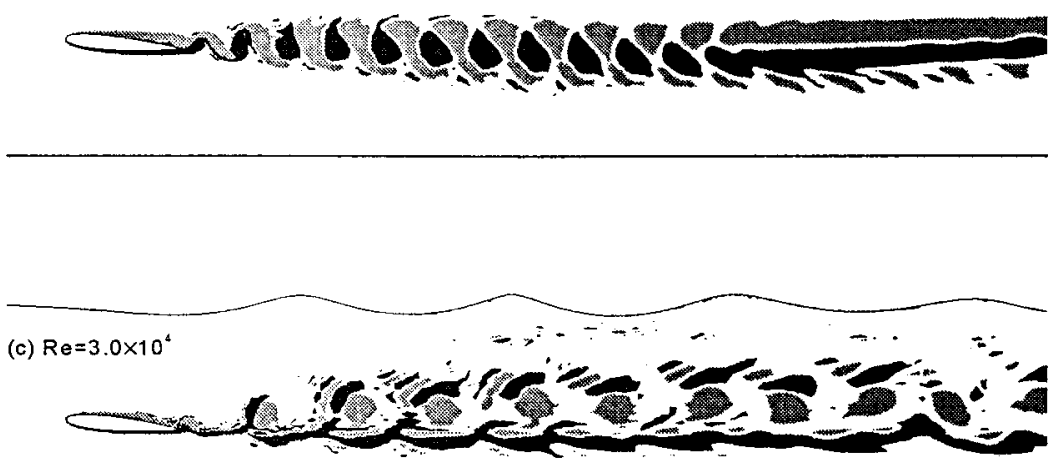

FIG. 7. Instantaneous vorticity contours at $t=26.0$ and Fr $=0.5672$ (color: $\omega^{-}=$light, $\omega^{+}=$dark, $|\omega| \geqslant 0.5$ ). 

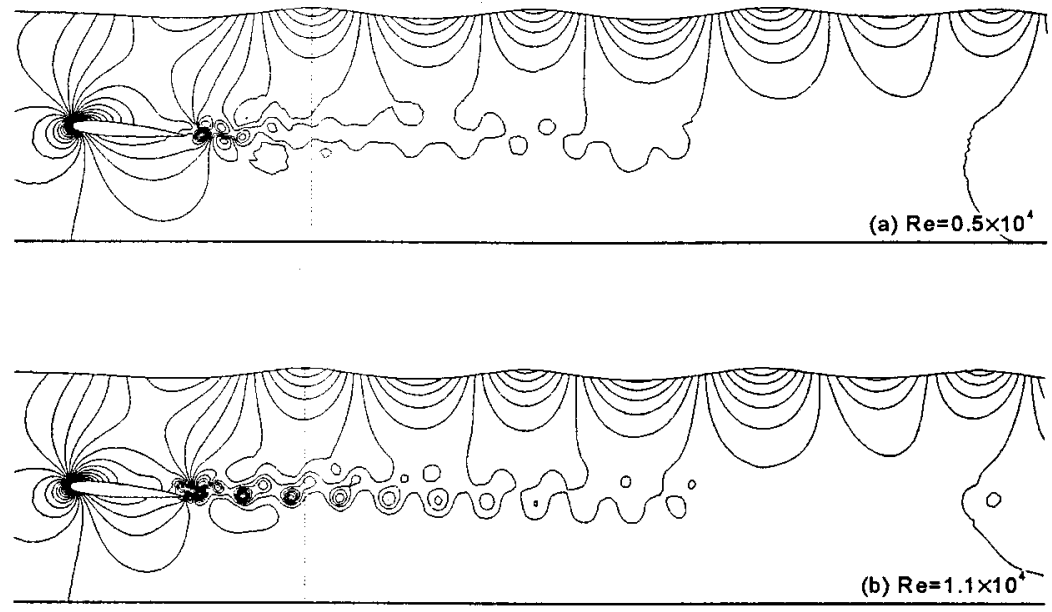

FIG. 8. Instantaneous pressure contours at $t=26.0$ and $\mathrm{Fr}=0.5672\left(P_{\min }=-0.5, P_{\max }=0.5, \Delta P=0.025\right)$.

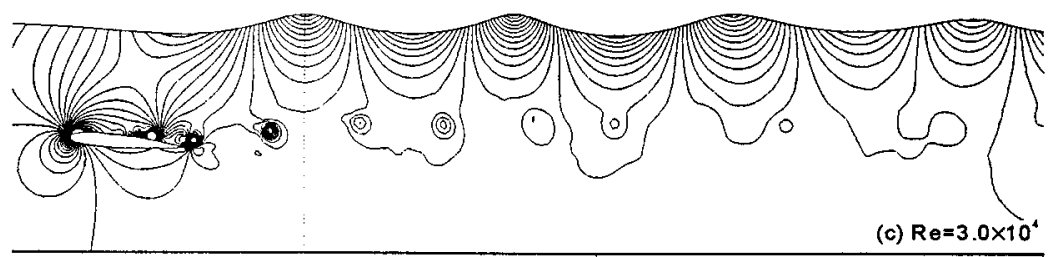

cooperative system with the undergoing trailing wake. As pointed out by Shen et al., ${ }^{17}$ such a surface-layer is caused by the zero tangential stress condition at the free surface. The wake structures, such as the velocity fluctuation, can also be seen in these figures. It is noted that the maximum streamwise velocity takes place at the contact points of a pair of counter-rotating vortices where the vorticity is cancelled. The lowest pressure spot for each case corresponds to the negative vortex core that is ascending with increasing Re.

\section{Free-surface effect on trailing vortices}

The free-surface effect on the trailing vortices is studied by analyzing the dependence of the frequency at which vortices are shed in a Kármán vortex street. Velocity signals are recorded at the sampling points of $1.5 \mathrm{~L}$ and $2.5 \mathrm{~L}$ in the downstream horizontal to the trailing edge. A large number of cases are computed and the Strouhal numbers are obtained by spectral analysis using the Fourier transformation of the
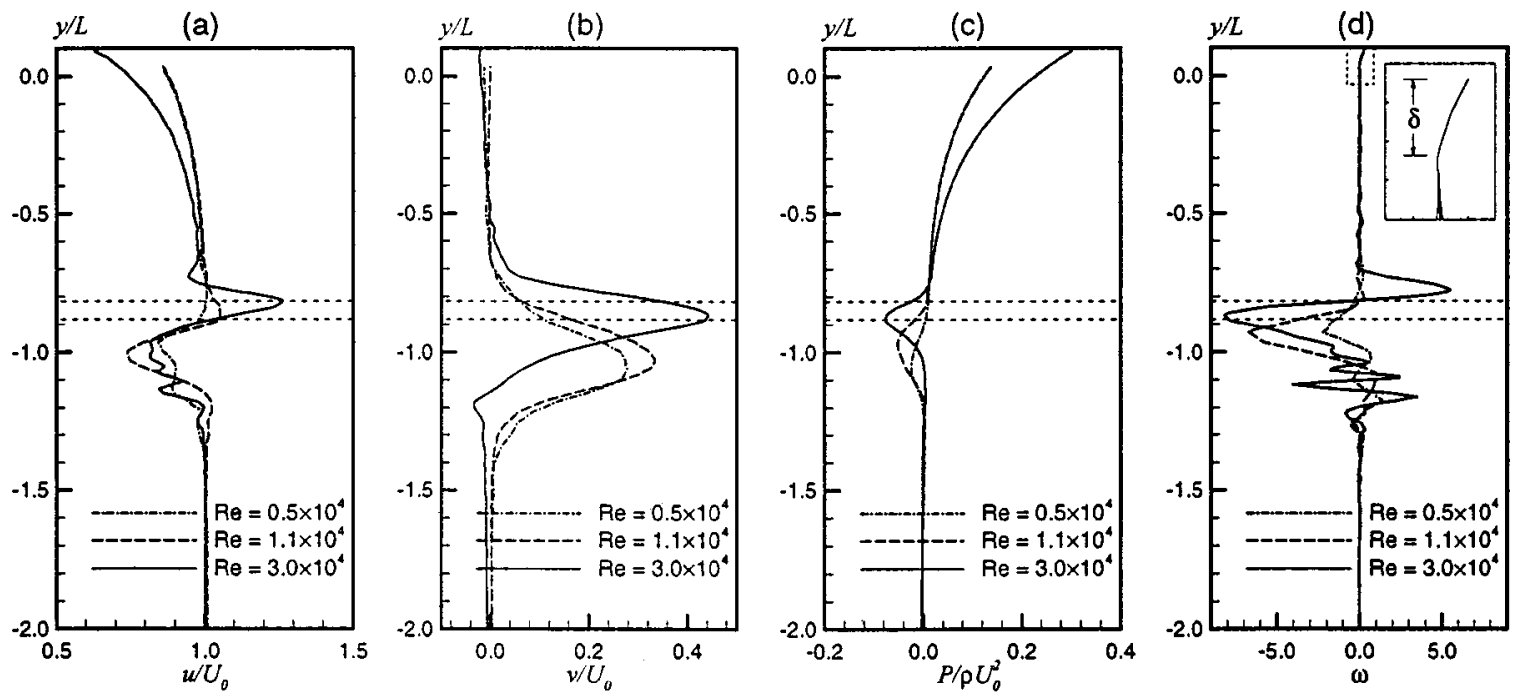

FIG. 9. Computed field variables probed along a vertical line through the first wave crest (as indicated in the flow field by dashed lines in Fig. 8) for $t$ $=26.0$ and $\mathrm{Fr}=0.5672$ : (a) velocity $u$, (b) velocity $v$, (c) pressure $P$, and (d) vorticity $\omega$. 


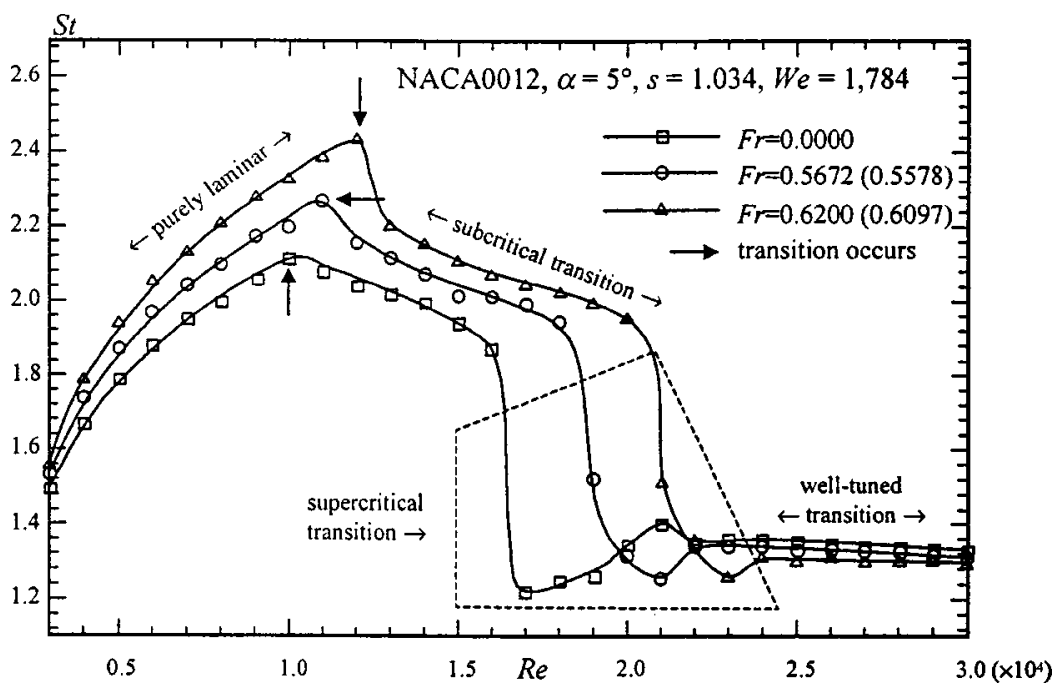

FIG. 10. The vortex-shedding Strouhal number vs the Reynolds number for three different Froude numbers obtained from the power spectra of wake velocity signals.

wake velocity fluctuations (frequency accuracy $\Delta f=0.01$, time integration interval $t=12.0-26.0$ ). Figure 10 shows the vortex-shedding St versus Re for three different Froude numbers. Four flow stages can be distinguished, namely the purely laminar stage, the subcritical transition stage, the supercritical transition stage and the well-tuned transition stage. Some interesting and unique characteristics are revealed for the trailing-vortex development beneath a free surface.

For lower Reynolds numbers when the flow remains purely laminar, predominant vortex-shedding Strouhal frequencies $f_{s}$ are detected with their harmonics $\left(m f_{s}\right)$ in the spectra. The Strouhal number increases with Re and matches very well the St-Re relationship suggested by Williamson ${ }^{15}$ based on experiments of laminar flows past a circular cylinder without a free surface,

$$
\mathrm{St}=A_{1} / \operatorname{Re}+A_{2}+A_{3} \operatorname{Re} .
$$

However, the original coefficients $A_{1}=-3.3265, A_{2}$ $=0.1816$, and $A_{3}=1.6 \times 10^{-4}$ need be modified due to the change of geometry, and are apparently functions of Fr in the present free-surface flow. After data analysis, we propose a $\mathrm{St}-\mathrm{Re}-\mathrm{Fr}$ relationship for the vortex shedding behind a NACA0012 hydrofoil in a free-surface flow, in which coefficients $A_{i}(i=1-3)$ are revised empirically by

$$
A_{i}=B_{i} \exp \left(\beta \operatorname{Fr}_{s}^{\alpha}\right)+C_{i} \exp \left(\beta \operatorname{Fr}_{s}^{-\alpha}\right),
$$

with $\alpha=6, \beta=-0.052$. Regression by the least-squares method produces coefficients $B_{i}$ and $C_{i}$ as listed in Table I. To be more representative of the flow physics, the submergence-based Froude number $\mathrm{Fr}_{s}$ is adopted. Figures 11(a) and 11(b) compare the proposed formula with the computational data for isolated Froude and Reynolds numbers,

TABLE I. Coefficients $B_{i}$ and $C_{i}$ for a NACA0012 hydrofoil in a freesurface flow.

\begin{tabular}{cccr}
\hline \hline$i$ & 1 & 2 & 3 \\
\hline$B_{i}$ & -1688.142 & 1.986 & $2.886 \times 10^{-5}$ \\
$C_{i}$ & -2336.763 & 0.855 & $-4.507 \times 10^{-6}$ \\
\hline \hline
\end{tabular}

respectively. Additional computations are carried out for other Froude numbers to cross-verify this relationship. The resultant relative mean accuracy of the proposed formula is

$$
\epsilon=\left[\Sigma\left(\mathrm{St}-\mathrm{St}_{\text {comput }}\right)^{2} / \Sigma \mathrm{St}^{2}\right]^{1 / 2}=4.5 \times 10^{-3},
$$

for all the computations shown in Figs. 11(a) and 11(b). It is worth to mention that formulas (19) and (20) are only valid for purely laminar flows and no breaking waves on the free surface.

The dependence of $A_{i}$ on Fr strongly exhibits the correlation of the viscosity and gravity in the shed trailing vortices. However, the differences among St curves for various Froude numbers are not so remarkable for lower Reynolds numbers. It is evident that the free surface has less impact on the large-scale vortex alternation in flows with relatively high viscosity. It is of particular interest to note from Fig. 11(a) that the three St-Re curves cross each other at a critical $\operatorname{Re} \cong 2850$ [see the dotted circle in Fig. 11(a)]. By extrapolation, one may also obtain the minimum Re at which regular Kármán street can be observed $(\mathrm{Re} \cong 850,1000$, and 1100 for $\mathrm{Fr}_{s}=0.0,0.5578$, and 0.6097, respectively, with St $=0.0$ ). This explains a very important scenario of the freesurface flow that a higher Fr will result in a later onset of vortex shedding. This conjecture is strongly supported by the physical experiments of Sheridan, Lin, and Rockwell. ${ }^{1}$ They found that the presence of a free surface allows small-scale Kelvin-Helmholtz instabilities to occur, but inhibits the onset of Kármán instability. However, once the Kármán instability has been triggered and Re has exceeded this critical value, it is clearly shown from the present computational results that a higher Fr will always lead to a faster vortexshedding frequency in laminar flows.

In addition, remarks can be drawn readily from the $\mathrm{St}-\mathrm{Fr}_{s}$ curves in Fig. 11(b) about the Froude-number effect on the shedding frequency. As far as the vortex alternation is concerned, the free-surface effect can virtually be ignored for all Reynolds numbers with $\mathrm{Fr}_{s} \leqslant 0.45$. For $0.5 \leqslant \mathrm{Fr}_{s} \leqslant 0.75, \mathrm{St}$ is almost linearly proportional to $\mathrm{Fr}_{s}$. By dropping the first term in (12), the slope $k$ can be roughly calculated from (19) and (20) as 

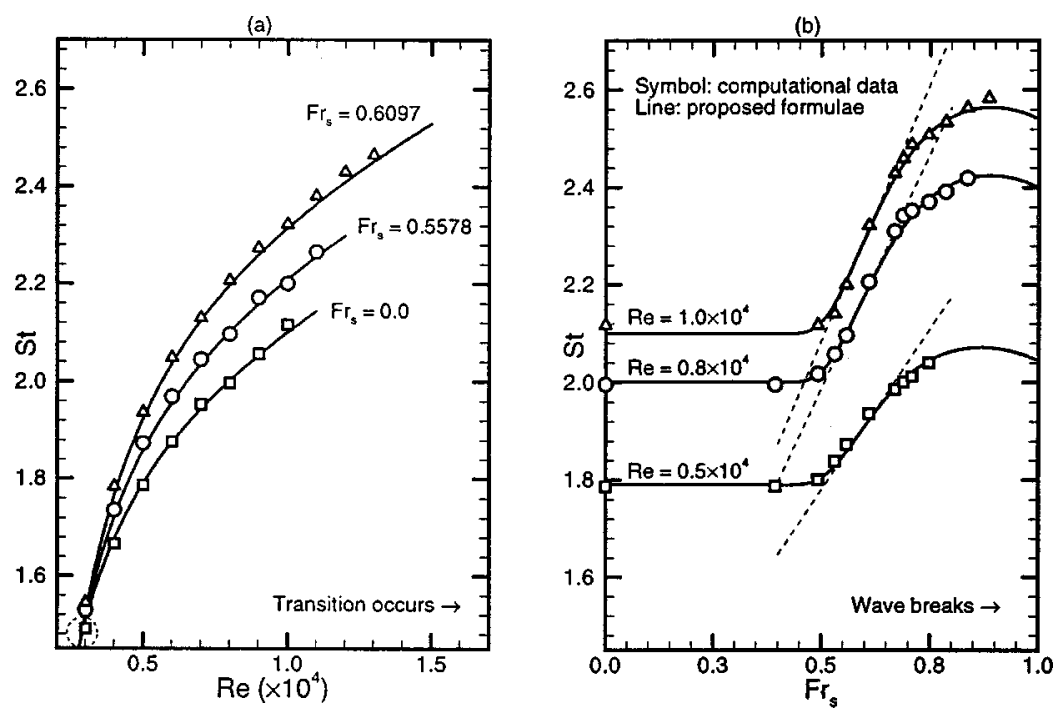

FIG. 11. Strouhal-Reynolds-Froude number relationship for the laminar vortex shedding behind a NACA0012 hydrofoil in a free-surface flow (We $=1784, s=1.034, \alpha=5^{\circ}$ ).

$$
k \cong C(1+\alpha) \exp (-1 / \alpha-1) / \mathrm{Fr}^{*} \cong 3.66 C,
$$

where

$$
C(\mathrm{Re})=C_{1} / \mathrm{Re}+C_{2}+C_{3} \operatorname{Re},
$$

and $\mathrm{Fr}^{*}$ denotes the turning point of the $\mathrm{St}-\mathrm{Fr}$ curves at which the second derivative vanishes

$$
\mathrm{Fr}^{*}=\left(\frac{-\alpha \beta}{1+\alpha}\right)^{1 / \alpha} \cong 0.5954 \text {. }
$$

The tangent lines are shown in Fig. 11(b) by the dotted lines for relevant Reynolds numbers. It is noted that $k$ increases slightly with increasing Re but is independent of Fr. Equation (20) indicates that the free-surface effect will also disappear as $\mathrm{Fr}_{s} \rightarrow \infty$ yet the flow remains purely laminar and no wave breaks (though these requirements can hardly be met in the present problem). The present formulas also indicate that after $\mathrm{Fr}_{s}$ exceeds a threshold value, St starts to decrease as expected, and converges to a lower level than that for the $\mathrm{Fr}_{s}=0$ case.

Let us discuss Fig. 10 again. At the early stage of transition, the shedding frequency reaches a threshold at a moderate $\mathrm{Re}$, and then drastically downshifts, indicating that the transition has been triggered. Such a scenario is presumably related to the formation and separation of the second vortex $\left(e_{2}\right)$ and the strong nonlinear interaction with the primary one $\left(e_{1}\right)$ as shown in Fig. 6. Persillon and Braza ${ }^{18}$ numerically confirmed the sudden downshift or the so-called discontinuity of Strouhal frequency in a region between $\mathrm{Re}$ $=180-260$ in the wake of a circular cylinder. Comparing with the instability of $2 \mathrm{D}$ calculations, they conjectured that such a transition feature is strictly due to the 3D effect caused by the energy exchange between the vortex shedding motion and the generation of fine-scale turbulent motion. Nevertheless, the present frequency downshift for a hydrofoil is strikingly different from that of a circular cylinder in the sense that it exists in a 2D flow and is under the strong influence of a free surface. The frequency downshifting might be regarded as a subcritical and supercritical two-step process after the threshold value has been reached. The downshift of the shedding frequency becomes steep or even discontinuous with increasing Fr. This two-step discontinuity is unique to, or at least amplified by, the free-surface wave motion. It is also observed that the dependence of St on Re is not so evident during this stage.

The occurrence of the substantial frequency drop is delayed as Fr increases (as marked by arrows in Fig. 10). This verifies that the presence of a free surface suppresses the formation and growth of the secondary vortex $\left(e_{2}\right)$. Re at which the transition occurs is approximately $1.0 \times 10^{4}, 1.1$ $\times 10^{4}$, and $1.3 \times 10^{4}$ for $\mathrm{Fr}=0.0,0.5672$, and 0.62 , respectively. The delayed transition, from the point view of energy, is presumably owing to the amplification of the energy transformation from the kinetic form to the potential one. After the transition has occurred, the vortex structure and the power spectra become rather complicated (see Fig. 12 for the flow structure and Fig. 13 for a comparison of velocity fluctuations at three typical flow stages), especially at high Froude numbers. It is noted that the Strouhal numbers as shown in Fig. 10 for this stage are hard to discern from the spectra and may not be so reliable, since a broad range of irregular and metastable low frequencies with high energy levels are excited at this initial transition stage. This intermittent state and vortex-shedding deceleration-cessation are similar to the experimental findings of Bearman and Zdravkovich $^{19}$ in a flow past a horizontal circular cylinder very close to (almost touching) a plane boundary parallel to the free stream. There exists a similarity of trailing-wake structures between a body close to a wall and a fully submerged body adjacent to a free surface, in the sense of block-

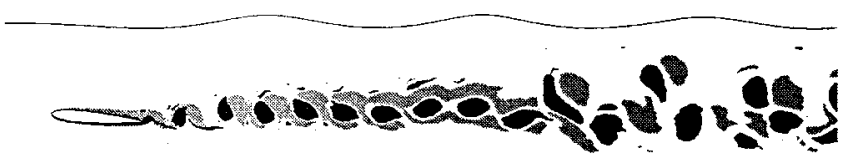

FIG. 12. Instantaneous vorticity contours when the Strouhal frequency downshifts at $t=26.0, \mathrm{Fr}=0.62$, and $\mathrm{Re}=2 \times 10^{4}$ (color: $\omega^{-}=$light, $\omega^{+}$ $=$ dark, $|\omega| \geqslant 0.5$ ). 
(a) $\mathrm{Re}=1.0 \times 10^{4}$, purely laminar

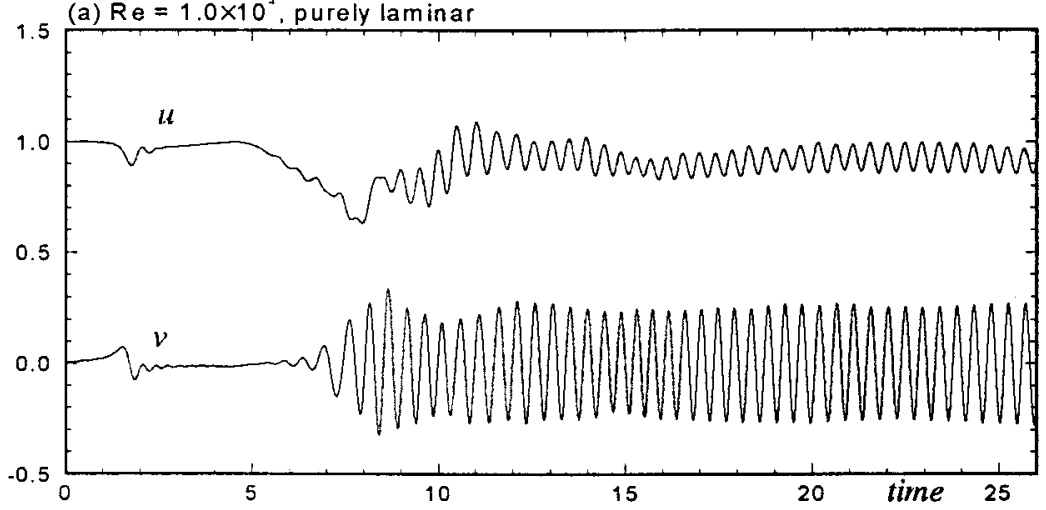

(b) $\operatorname{Re}=2.0 \times 10^{4}$, metastable transition

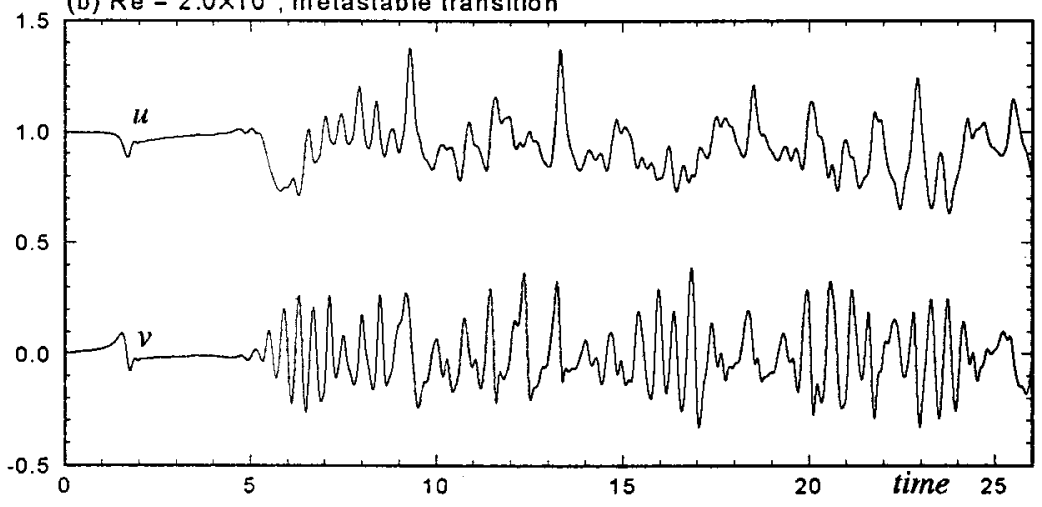

(c) $\mathrm{Re}=3.0 \times 10^{4}$, well-tuned transition

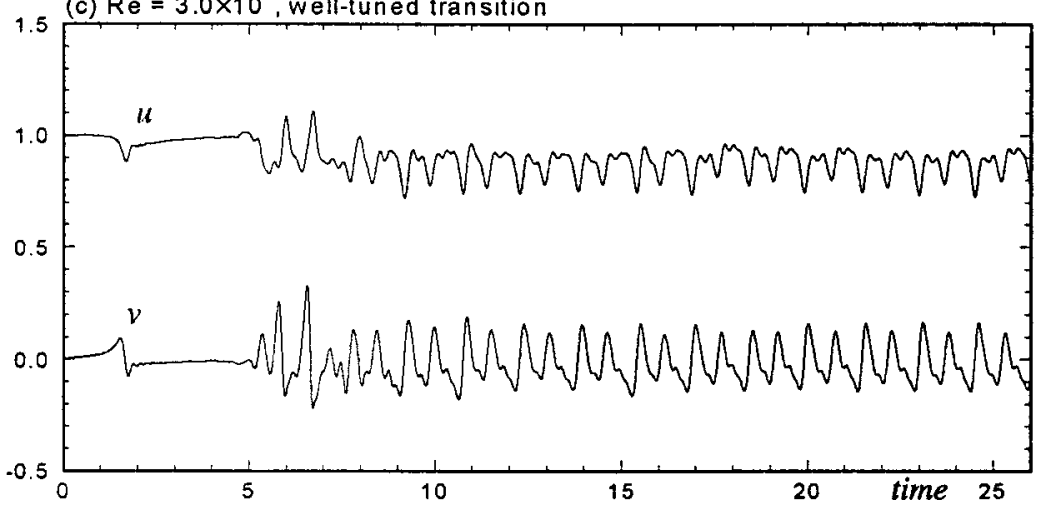

FIG. 13. Velocity signals recorded at a sampling point $x=1.5 \mathrm{~L}$ downstream horizontal to the trailing edge at different flow stages for $\mathrm{Fr}=0.5672$. age that a free surface is indeed a material boundary. Nevertheless, the curvature of, and the free traction at, the free surface makes the latter scenario even more complicated. Further meticulous physical experiments are definitely needed to confirm this chaotic behavior and the excitation mechanism influenced by the free surface. The differences among the shedding frequencies at various Froude numbers strongly indicate a correlation between viscosity and gravity effects.

Surprisingly, the Strouhal-shedding oscillation approaches a relatively stable stage after the deceleration for all the Froude numbers computed. Meanwhile, a distinct transition (or the so-called secondary) frequency $f_{t}$ can be identified from the power spectra, being suggestive of the secondary vortex characteristics. The secondary vortices and the primary Strouhal vortices are both originated from the same separated shear layer. However, this transition frequency, as first observed by Bloor ${ }^{20}$ from a cylinder wake flow, is apparently unrelated to the periodic Strouhal vortex-shedding process, and contributes to the transition of the Kármán vortex street. Figures 14(a)-14(c), on a log-plot, show typically the power spectra of wake velocity fluctuations at $\mathrm{Re}=3.0$ $\times 10^{4}$ for $\mathrm{Fr}=0.0,0.5672$, and 0.62 , respectively. It is evident that the well-tuned spectra consist of $f_{s}$ and $f_{t}$, their harmonic frequencies $\left(m f_{s}, n f_{t}\right)$ and their combinations $\left(m f_{s} \pm n f_{t}\right)$. For every given set of conditions, the resulting $f_{t}$ lies in between $2 f_{s}$ and $3 f_{s}$. The shedding frequency $f_{s}$ seems to be "locked," slowly varying with respect to the Reynolds and Froude numbers computed. Again, this vortex behavior is quite comparable to the observations of Bearman and Zdravkovich ${ }^{19}$ in a flow past a circular cylinder near a plate, in which the large-scale Kármán instability is remarkably similar and $\mathrm{St}$ is almost invariant with respect to the gap ratio $(G / D)$ of the cylinder-plate distance to the cylinder 

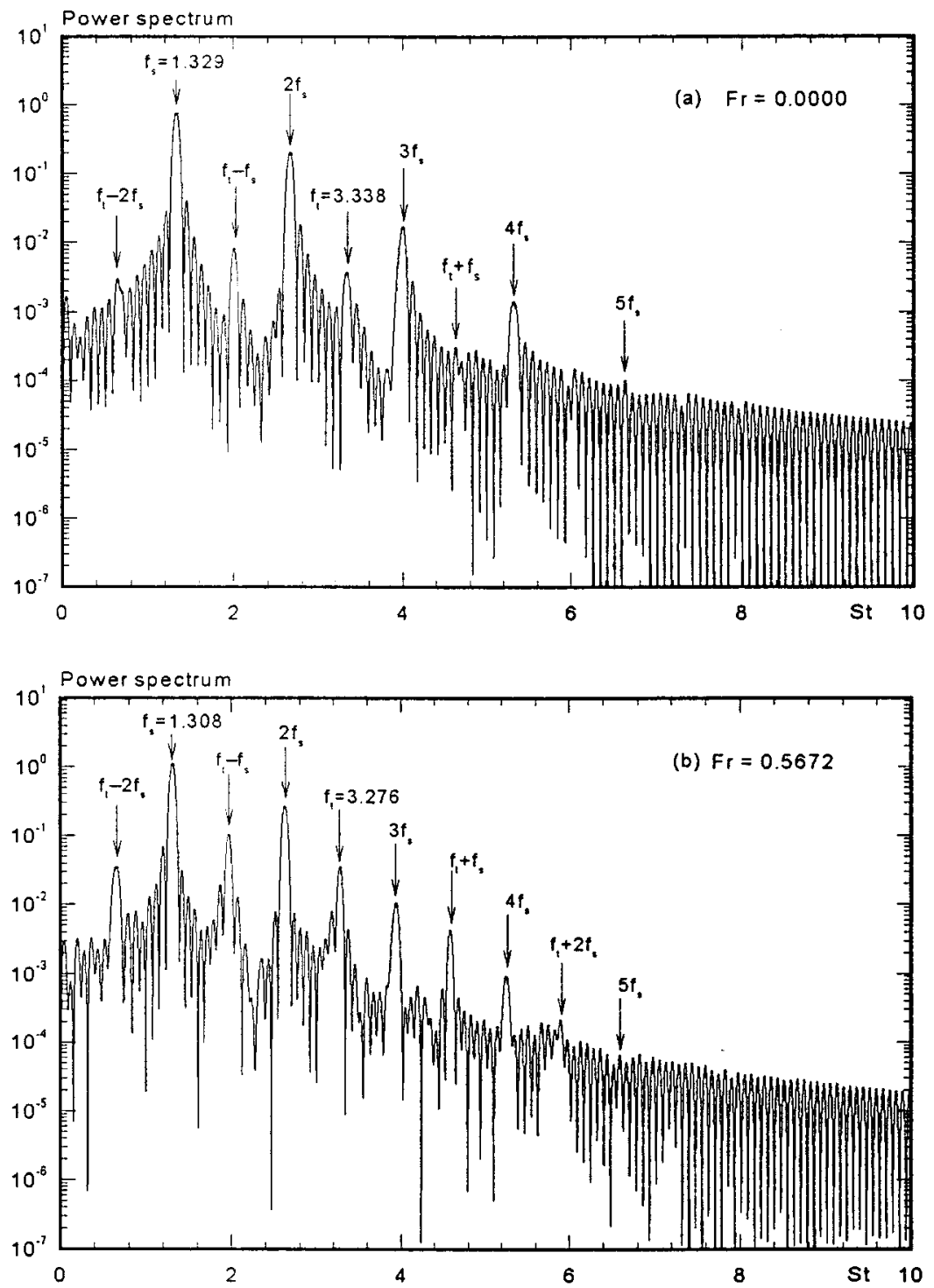

FIG. 14. Power spectra of velocity signals recorded at a sampling point $x=1.5 \mathrm{~L}$ downstream horizontal to the trailing edge for $\operatorname{Re}=3 \times 10^{4}$.

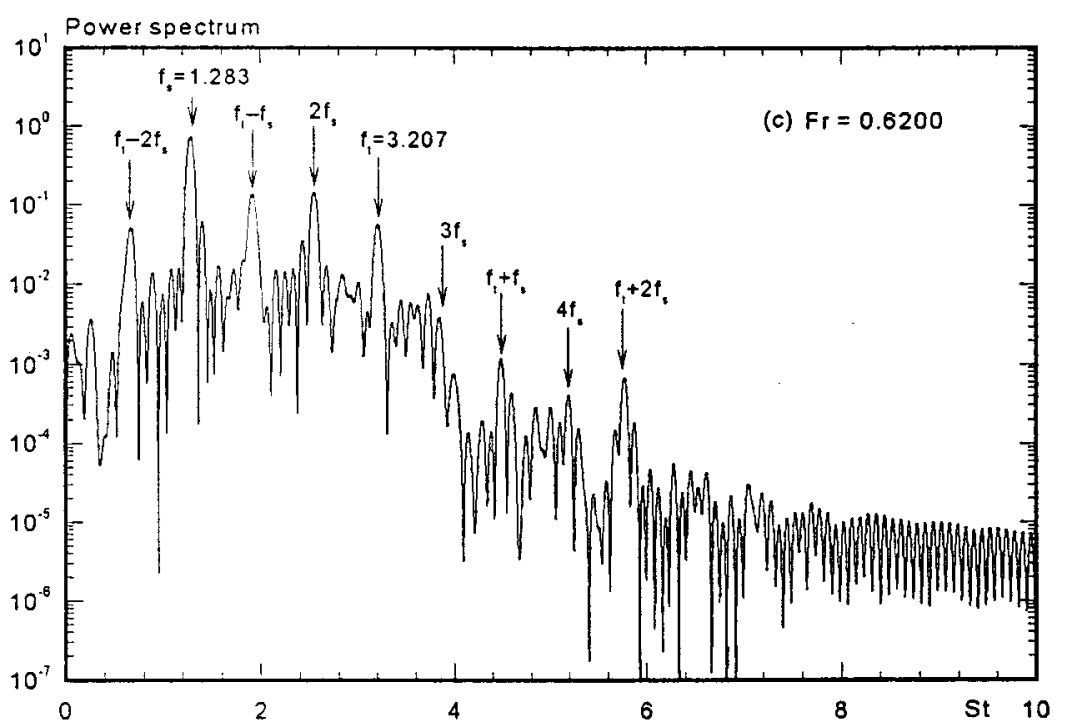


TABLE II. Strouhal/transition frequencies at various Froude numbers, Re $=3 \times 10^{4}$ and $\mathrm{We}=1784$.

\begin{tabular}{llll}
\hline \hline $\mathrm{Fr}$ & 0.0000 & 0.5672 & 0.6200 \\
$f_{s}$ & 1.329 & 1.308 & 1.285 \\
$f_{t}$ & 3.338 & 3.276 & 3.207 \\
\hline \hline
\end{tabular}

diameter for $G / D>0.3$. For fixed Re, a higher Fr will induce a slightly lower transition frequency (see Table II). The presence of a free surface, acting as a kinematic cushion effect, delays-suppresses the transition process, although the largescale wake alternation is indeed accelerated by higher Froude numbers when the flow is still purely laminar.

Having observed the apparent similarity between the structures in cylinder wake flows and free-shear flows, Bloor $^{20}$ first suggested that there should exist a universal transition mechanism. Experiments have shown that, for a flow past a circular cylinder, the transitional frequency (normalized by the vortex-shedding frequency) follows a powerlaw relation in terms of Re based on cylinder diameter ${ }^{20}$

$$
f_{t} / f_{s}=\left(\operatorname{Re}_{d} / 470\right)^{\chi}
$$

where index $\chi=0.5$ was further revised to 0.87 by Wei and Smith $^{21}$ via physical experiments and numerically confirmed later by Ling et al. ${ }^{22}$ Therefore, we have reason to speculate that a similar relationship for the fully submerged hydrofoil in a free-surface flow may also exist and apparently dependent on the gravity as well. Due to the free-surface suppressing effect on transition, we may further expect that the positive slope of $\chi \sim \log \left(f_{t} / f_{s}\right)$ will be slightly reduced with respect to the submergence-based Froude number $\mathrm{Fr}_{s}$. This conjecture probably directs the future work towards freesurface turbulent flows.

\section{CONCLUSIONS}

A finite element method is formulated in this paper that has the flexibility to handle not only the arbitrary geometrical configurations but also the unique natural boundary integrals imposing on the free-surface boundaries. Parametric study in the present numerical experiments has preliminarily revealed some interesting and unique effect of the free surface on the trailing vortices. The presence of a free surface increases the vortex-alternating frequency. The vortex street demonstrates quite different shedding patterns with increasing Re. An empirical St-Re-Fr relationship for the vortex shedding in a free-surface flow is proposed for $\operatorname{Re} \leqslant 1 \times 10^{4}$. The freesurface vortex forms a thin free-surface viscous sublayer and a cooperative system with the undergoing trailing wake. An initial transition stage is identified between the purely laminar stage and the fine-tuned transition stage. The flow transition occurs as the secondary vortex emerges on the suction side of a hydrofoil. After reaching a peak value, the vortexshedding frequency experiences a subcritical and supercritical two-step drastic downshift or even a discontinuity as the secondary vortex grows. An independent and initially metastable transition frequency gets stronger and discernable. The power spectra of the vortex street consist of harmonics of the Strouhal-vortex shedding frequency, the transition frequency and their combinations, suggesting a strong nonlinear inter- action between the primary and secondary vortices. Meanwhile, the large-scale Strouhal-vortex alternation seems to be "locked," slowly varying with respect to $\mathrm{Fr}$ and high $\mathrm{Re}$ computed. The free-surface effect, acting on the large-scale vortex alternations, is reflected on the secondary flow structure as well. The vortex-street behavior is very much dependent on the secondary transition features. It is found that the presence of a free surface inhibits the onset of Kármán instability, suppresses secondary transition activities, and delays the undergoing transition process. This is presumably due to the transfer of kinetic energy to potential energy in the presence of a free surface.

\section{ACKNOWLEDGMENTS}

This research was sponsored by the Hong Kong Research Grants Council under Grants No. HKU 7066/97E and No. NSFC/HKU 8.

${ }^{1}$ J. Sheridan, J. C. Lin, and D. Rockwell, "Flow past a cylinder close to a free surface," J. Fluid Mech. 330, 1 (1997).

${ }^{2}$ T. Sarpkaya, "Vorticity, free surface and surfactants," Annu. Rev. Fluid Mech. 28, 83 (1996).

${ }^{3}$ S. Ohring and H. J. Lugt, "Interaction of a viscous vortex pair with a free surface," J. Fluid Mech. 227, 47 (1991).

${ }^{4}$ W. T. Tsai and D. K. P. Yue, "Computation of nonlinear free-surface flows," Annu. Rev. Fluid Mech. 28, 249 (1996).

${ }^{5}$ C. Zhang, L. Shen, and D. K. P. Yue, "The mechanism of vortex connection at a free-surface," J. Fluid Mech. 384, 207 (1999).

${ }^{6}$ B. R. Hodges and R. L. Street, "On simulation of turbulent nonlinear free-surface flows," J. Comput. Phys. 151, 425 (1999).

${ }^{7}$ D. H. Zhang and A. T. Chwang, "On solitary waves forced by underwater moving objects," J. Fluid Mech. 389, 119 (1999).

${ }^{8}$ T. Chen, A. T. Chwang, and D. H. Zhang, "Large-eddy simulations for a free-surface turbulent flow past vertical cylinders," Proceedings of the 10th International Offshore and Polar Engineering Conference, Seattle, WA 3, 60 (2000).

${ }^{9}$ M. Longuet-Higgins, "Capillary rollers and bores," J. Fluid Mech. 240, 659 (1992).

${ }^{10}$ P. M. Gresho and R. L. Sani, Incompressible Flow and the Finite Element Method (Wiley, New York, 1998).

${ }^{11}$ M. Israeli and S. A. Orszag, "Approximation of radiation boundary conditions,” J. Comput. Phys. 41, 115 (1981).

${ }^{12}$ T. Chen and A. T. Chwang, "Calculations of nonlinear waves generated by complex body motion," J. Mech. Eng. Sci. Part C 214, 771 (2000).

${ }^{13}$ A. Lungu and K. Mori, "A study on numerical schemes for more accurate and efficient computations of free-surface flows by finite difference method," J. Society Naval Architects Japan 173, 9 (1993).

${ }^{14}$ U. Ghia, K. N. Ghia, and C. T. Shin, "High-Re solution for incompressible flow using the Navier-Stokes equations and a multigrid method,' J. Comput. Phys. 48, 387 (1982).

${ }^{15}$ C. H. K. Williamson, "Defining a universal and continuous StrouhalReynolds number relationship for the laminar vortex shedding of a circular cylinder," Phys. Fluids 31, 2742 (1988).

${ }^{16}$ J. H. Duncan, "The breaking and non-breaking wave resistance of a twodimensional hydrofoil," J. Fluid Mech. 126, 507 (1983).

${ }^{17}$ L. Shen, X. Zhang, D. K. P. Yue, and G. S. Triantafyllon, "The surface layer for free-surface turbulent flows," J. Fluid Mech. 386, 167 (1999).

${ }^{18} \mathrm{H}$. Persillon and M. Braza, "Physical analysis of the transition to turbulence in the wake of a circular cylinder by three-dimensional NavierStokes simulation," J. Fluid Mech. 365, 23 (1998).

${ }^{19}$ P. W. Bearman and M. M. Zdravkovich, "Flow around a circular cylinder near a plane boundary," J. Fluid Mech. 89, 33 (1978).

${ }^{20}$ M. S. Bloor, "The transition to turbulence in the wake of a circular cylinder," J. Fluid Mech. 19, 290 (1964).

${ }^{21}$ T. Wei and C. R. Smith, "Secondary vortices in the wake of circular cylinders," J. Fluid Mech. 169, 513 (1986).

${ }^{22}$ G. C. Ling, A. T. Chwang, J. Y. Niu, and D. L. Wang, "Numerical study of the near-wake flow transition characteristics and nonlinear interaction," Sci. China, Ser. A: Math., Phys., Astron. 27, 439 (1997). 